\title{
Efeito do tratamento térmico na concentração de carotenóides, compostos fenólicos, ácido ascórbico e capacidade antioxidante do suco de tangerina murcote
}

Effect of thermal treatment on the carotenoid, phenolic compound and ascorbic acid concentrations, and the antioxidant capacity of murcott tangerine juice

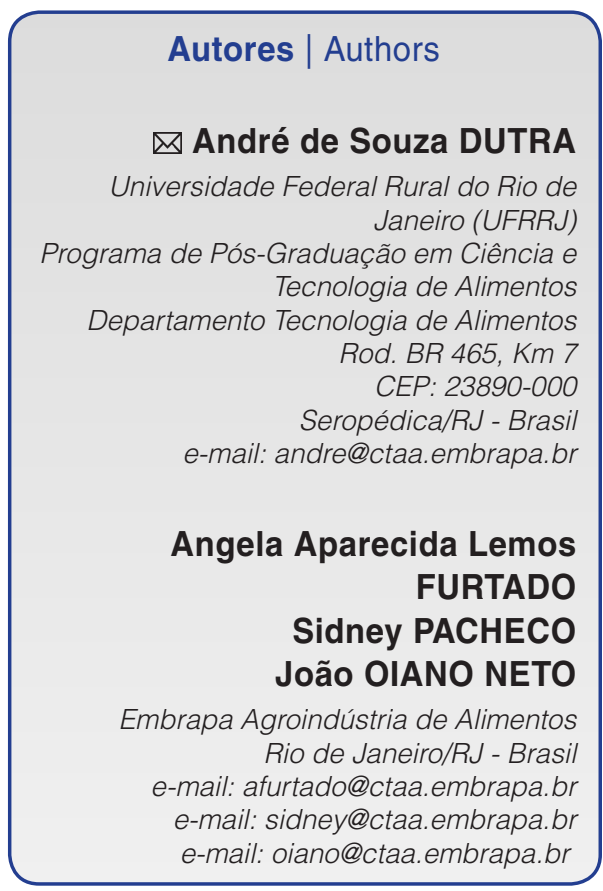

Autor Correspondente / Corresponding Author

Recebido / Received: 03/05/2010 Aprovado / Approved: 21/03/2012 Publicado / Published: set./2012

\section{Resumo}

Neste estudo, avaliou-se o efeito do tratamento térmico do suco de tangerina murcote em relação ao teor de carotenóides totais, ao perfil de carotenóides (luteína, zeaxantina, $\beta$-criptoxantina e $\beta$-caroteno), aos compostos fenólicos totais, ao ácido ascórbico e à capacidade antioxidante. No processo, utilizou-se um trocador de calor tubular, sendo avaliados 11 tratamentos seguindo um ensaio fatorial completo $2^{2}$ com três repetições no ponto central, com quatro pontos axiais e uma região de observação de 88 a $100{ }^{\circ} \mathrm{C}$ e 16 a 44 s. O teor de caratenóides totais decresceu de forma significativa em quase todos os tratamentos aplicados, se comparado ao valor encontrado para o suco in natura. A maioria dos tratamentos não reduziu de forma significativa os teores dos caratenóides luteína, zeaxantina e $\beta$-criptoxantina. Além disso, o teor de $\beta$-caroteno não sofreu alterações significativas em todos os tratamentos avaliados. A análise de variância mostrou que, para os caratenóides, não ocorreram interações significativas entre as variáveis temperatura e tempo. Os teores de ácido ascórbico e compostos fenólicos totais apresentaram reduções em seis condições avaliadas, ao passo que, em quatro, observou-se um aumento dos teores de compostos fenólicos totais, causado provavelmente pela evaporação da água presente no suco. A maior redução do teor de ácido ascórbico e o maior aumento para compostos fenólicos totais foi observado para o binômio $100^{\circ} \mathrm{C} / 30$ s. O teor de ácido ascórbico sofreu de forma significativa a influência da temperatura e esse processo de degradação pode ser descrito por meio de um modelo quadrático. Todos os tratamentos utilizados reduziram a capacidade antioxidante do suco, sendo mais intensa para o binômio $100{ }^{\circ} \mathrm{C} / 30 \mathrm{~s}$. As variações observadas não alteraram de maneira considerável as características nutricionais do suco e os tratamentos térmicos realizados a $94{ }^{\circ} \mathrm{C}$ por 16 a $44 \mathrm{~s}$ possibilitaram menores alterações e/ou maiores retenções nos compostos determinados.

Palavras-chave: Citrus; Tangerina murcote; Suco; Tratamento térmico; Capacidade antioxidante. 


\section{Summary}

This study evaluated the effect of thermal treatment on the total carotenoid concentration, the carotenoid profile (lutein, zeaxanthin, $\beta$-cryptoxanthin and $\beta$-carotene), total phenolic compound content, ascorbic acid content and antioxidant capacity of Murcott Tangerine juice. Using a tubular heat exchanger eleven treatments were evaluated based on a full $2^{2}$ factorial design with three replicates at the central point, four axial points and a region of observation from 88 to $100{ }^{\circ} \mathrm{C}$ and from 16 to $44 \mathrm{~s}$. The total carotenoid content decreased significantly in almost all treatments when compared with the results obtained for the fresh juice. The carotenoid levels (lutein, zeaxanthin and $\beta$-cryptoxanthin) were not significantly reduced by most of the treatments studied, and the $\beta$-carotene content was not significantly changed by any treatment. For the carotenoid contents, the analysis of variance showed no significant interactions between the variables of temperature and time. The levels of ascorbic acid and total phenolic compounds decreased in six of the conditions evaluated, while in four treatments an increase in the total phenolic compounds was observed, probably due to evaporation of water from the juice. The greatest reduction and the greatest increase of, respectively, ascorbic acid and total phenolic compounds were observed for the binomial of $100{ }^{\circ} \mathrm{C} / 30 \mathrm{~s}$. The ascorbic acid content was significantly influenced by the temperature, and its degradation process can be described using a quadratic model. All the treatments applied reduced the antioxidant capacity of the juice, but the reduction was higher for the binomial $100{ }^{\circ} \mathrm{C} / 30 \mathrm{~s}$. The nutritional characteristics of the juice were little affected by the changes observed, and the heat treatments carried out at $94{ }^{\circ} \mathrm{C}$ for 16 to $44 \mathrm{~s}$ allowed for the smallest changes and/or the highest retentions of the determined compounds.

Key words: Citrus; Murcott tangerine; Juice; Thermal treatment; Antioxidant capacity. 
Efeito do tratamento térmico na concentração de carotenoides, compostos fenólicos, ácido ascórbico e capacidade antioxidante do suco de tangerina murcote

DUTRA, A. S. et al.

\section{Introdução}

O gênero Citrus apresenta frutos com elevada importância comercial, como a tangerina murcote. Esta é uma antiga variedade de tangor (híbrido da tangerina) desenvolvida na Flórida e propagada inicialmente pelos citricultores Charles Murcott e J. W. Smith, ficando conhecida pelos nomes de Murcott e Smith. Atualmente, nos Estados Unidos, essa variedade possui o nome oficial de tangerina Honey e, no Brasil, é mais conhecida como tangerina murcote (AZEVEDO e PIO, 2002).

O Brasil é o maior produtor mundial de citros, sendo atualmente o quarto maior produtor de tangerina, após China, Espanha e Japão (CASER e AMARO, 2006).

No Brasil, as variedades poncã e murcote são as mais cultivadas, mas o plantio da variedade murcote tem aumentado nos últimos anos por causa do bom rendimento apresentado na produção do suco - aproximadamente $50 \%$ da massa do fruto - e por possuir características sensoriais importantes para o mercado, como a cor da polpa e o teor de açúcares (FIGUEIREDO et al., 2006).

Os frutos cítricos e seus derivados apresentam efeitos benéficos à saúde em função das suas propriedades nutricionais, além de serem fontes de substâncias antioxidantes, como ácido ascórbico, compostos fenólicos e caratenóides (CANO et al., 2008). O interesse pelos caratenóides justifica-se pela sua ação como pró-vitamina A e por seu potencial antioxidante (LEACH et al.,1998; OLSON, 1996), prevenindo assim o surgimento de doenças cardiovasculares (DUGO et al.,2008), a degeneração macular (SNODDERLY, 1995) e o câncer (OMONI e ALUKO, 2005). As espécies do gênero Citrus são consideradas uma das principais fontes naturais desses compostos (GOODNER et al., 2001).

$\mathrm{Na}$ indústria alimentícia, a pasteurização é o tratamento térmico mais empregado na conservação de sucos de frutas que apresentam $\mathrm{pH}$ igual ou menor que 4,5. Tradicionalmente, os sucos são pasteurizados a temperaturas variando entre 60 e $100{ }^{\circ} \mathrm{C}$, durante poucos segundos. Durante a pasteurização, ocorre uma grande transferência de energia para o alimento e, em alguns casos, pode haver o desenvolvimento de reações indesejáveis (RIVAS et al., 2006).

Atualmente, os consumidores tornaram-se mais exigentes e optam por alimentos com uma vida útil maior e com suas características sensoriais e nutricionais preservadas (RIVAS et al., 2006).

Dessa forma, este estudo buscou avaliar a influência do tratamento térmico na degradação da atividade antioxidante, dos compostos fenólicos totais, da vitamina $C$ e dos caratenóides, substâncias e ações relevantes do suco de tangerina murcote, pois não existem dados na literatura científica que descrevam os efeitos desse tratamento nas características químicas do suco de tangerina murcote focadas neste estudo.

\section{Material e métodos}

\subsection{Matéria-prima}

As tangerinas murcote foram adquiridas em setembro de 2008 no comércio varejista do município do Rio de Janeiro-RJ e armazenadas em câmaras de refrigeração a $8{ }^{\circ} \mathrm{C}$ durante as etapas do processamento.

\subsection{Processamento e obtenção do suco}

Inicialmente, os frutos foram selecionados, lavados, higienizados com solução de hipoclorito de sódio e, posteriormente, descascados. Em seguida, os frutos descascados e inteiros foram despolpados em um extrator de sucos modelo Bonina (Itametal, Brasil) com malha de 0,6 mm e carga de $300 \mathrm{~kg} \cdot \mathrm{h}^{-1}$. Na sequência, o suco foi centrifugado em uma centrífuga de cesto modelo SIZE 2 (International Equipment Company) e rotação de $406 \mathrm{~g}$ durante $15 \mathrm{~min}$.

\subsection{Tratamento térmico}

O suco obtido após a centrifugação foi submetido a 11 tratamentos, seguindo um ensaio fatorial completo $2^{2}$, com três repetições no ponto central e quatro pontos axiais. O tratamento térmico foi conduzido em uma região de observação de 88 a $100^{\circ} \mathrm{C}$ e 16 a 44 s, utilizando-se um trocador de calor tubular modelo FT25D SSHE (Armfield, England). Os sucos tratados termicamente e in natura foram mantidos refrigerados durante a realização das análises quantitativas, que foram efetuadas em triplicata.

\subsection{Determinação do teor total e do perfil dos caratenóides por espectrofotometria UV-visível e cromatografia líquida de alta eficiência (CLAE)}

Os padrões analíticos utilizados na análise cromatográfica (luteína, zeaxantina, $\beta$-criptoxantina e $\beta$-caroteno) foram extraídos e purificados em coluna cromatográfica aberta empacotada com celite $545^{\circledR}$ (Tedia, USA), utilizando-se as seguintes fontes vegetais: (1) luteína - espinafre; (2) zeaxantina - laranjinha-de-jardim (Solanum pseudocapsicum); (3) $\beta$-criptoxantina - caqui, e (4) $\beta$-caroteno - cenoura (PACHECO, 2009).

As amostras de suco foram maceradas com celite $545^{\circledR}$ (Tedia, USA), extraídas com acetona (Tedia, USA) gelada e filtradas em funil de placa porosa. Os caratenóides do extrato cetônico foram extraídos por partição líquido-líquido em funil de separação com éter de petróleo (Tedia, USA) e água ultrapura obtida em um equipamento Millipore Elix ${ }_{5} /$ Milli $\mathrm{Q}^{\circledR} \mathrm{A}_{10}$ Gradient (Millipore, USA). A fase aquosa foi descartada, o extrato etéreo foi saponificado com KOH 10\% em metanol (Tedia, USA), com agitação por $16 \mathrm{~h}$, à temperatura ambiente, e ao abrigo da luz. Os caratenóides livres, obtidos na 
Efeito do tratamento térmico na concentração de carotenoides, compostos fenólicos, ácido ascórbico e capacidade antioxidante do suco de tangerina murcote

DUTRA, A. S. et al.

saponificação, foram novamente extraídos em funil de separação por partição líquido-líquido com éter de petróleo e água (PACHECO, 2009).

O extrato etéreo foi filtrado sob leito de sulfato de sódio anidro e uma alíquota foi retirada e utilizada para quantificar o teor total de caratenóides. Nesta análise, utilizou-se um espectrofotômetro UV-visível Shimadzu ${ }^{\circledR}$ modelo UV-1800, com cubeta de quartzo com $1,0 \mathrm{~cm}$ de caminho ótico e leitura em comprimento de onda de 449 nm (PACHECO, 2009).

A análise do perfil de caratenóides foi realizada utilizando-se um sistema cromatográfico Waters Alliance ${ }^{\circledR}$ modelo 2695 equipado com detector de arranjo de fotodiodos Waters ${ }^{\circledR}$ 2996, varredura de 350 a $600 \mathrm{~nm}$, coluna de fase reversa $\mathrm{C}_{30} \mathrm{YCM}^{\circledR}$ Carotenoid $(250 \times 4,6 \mathrm{~mm}, 3 \mu \mathrm{m})$ a $30^{\circ} \mathrm{C}$, fase móvel metanol: éter metil-terc-butílico 80:20 (v/v) (Tedia, USA) com eluição gradiente, fluxo 0,8 mL. $\mathrm{min}^{-1}$, com padronização externa e quantificação em triplicata (PACHECO, 2009).

\subsection{Determinação dos compostos fenólicos totais}

$\mathrm{Na}$ quantificação dos compostos fenólicos totais, utilizou-se o método espectrofotométrico proposto por Singleton e Rossi Junior (1965) e modificado por Georgé et al. (2005). O suco de tangerina foi extraído com acetona (Tedia, USA) 70\% durante $30 \mathrm{~min}$, filtrado em papel de filtro quantitativo e diluído com água destilada na razão de 1:10 (v/v).

O extrato obtido nessa etapa foi submetido à extração em fase sólida em cartucho Waters Oasis ${ }^{\circledR}$ HLB (fase estacionária copolímero de poli-[divinilbenzeno$N$-vinilpirrolidona]) para remoção dos compostos interferentes solúveis em água (açúcares redutores e ácido ascórbico). O extrato final foi submetido à reação com a solução de Folin-Ciocalteu em meio alcalino, com posterior leitura da absorvância em 760 nm, sendo os resultados expressos em miligrama de ácido gálico. $100 \mathrm{~g}^{-1}$ de amostra.

\subsection{Determinação da Vitamina C}

Adotou-se a metodologia descrita por Horwitz (2000) e modificada por Benassi e Antunes (1998). A padronização foi realizada com ácido ascórbico (SigmaAldrich, USA) transferindo-se 0,015 g do padrão para um balão volumétrico de $50 \mathrm{~mL}$ e completando-se o volume com solução de ácido oxálico (Sigma-Aldrich, USA) 2\%. Uma alíquota de $1,0 \mathrm{~mL}$ dessa solução foi transferida para um Erlenmeyer contendo $50,0 \mathrm{~mL}$ de ácido oxálico $2 \%$ e titulada com solução de 2,6-diclorofenolindofenol (Sigma-Aldrich, USA) 0,01\% até obter-se uma coloração rosa clara.

Para determinação do teor de ácido ascórbico, 2,5 $\mathrm{mL}$ do suco de tangerina foram transferidos para um balão volumétrico de $100,0 \mathrm{~mL}$ e o volume completado com ácido oxálico 2\%. Após 15 min sob agitação, uma alíquota de 2,0 $\mathrm{mL}$ do extrato foi transferida para um Erlenmeyer contendo $50,0 \mathrm{~mL}$ de ácido oxálico $2 \%$ e a solução titulada com 2,6-diclorofenolindofenol 0,01\% até obter-se uma coloração rosa clara. Os resultados foram expressos como miligramas de ácido ascórbico.100 g-1 da amostra.

\subsection{Determinação da atividade antioxidante}

Na determinação da atividade antioxidante, os extratos foram obtidos de acordo com a metodologia descrita por Rufino et al. (2007) e a quantificação foi realizada de acordo com a metodologia reportada por Re et al. (1999).

A determinação da atividade antioxidante foi feita pelo método espectrofotométrico baseado na captura do radical livre ABTS'+ [2,2'-azinobis-(3-etilbenzotiazolina)-6sulfonato]. O radical ABTS + foi gerado por meio da reação da solução aquosa de ABTS (Sigma-Aldrich, USA) 7,0 mM com solução de persulfato de potássio (Sigma-Aldrich, USA) $140,0 \mathrm{mM}$.

A solução foi mantida ao abrigo da luz por $14 \mathrm{~h}$ e diluída em seguida com água até obter-se uma absorvância de 0,7 $\pm 0,02$ a $734 \mathrm{~nm}$. Uma amostra contendo uma massa aproximada de $3,0 \mathrm{~g}$ foi extraída com $10 \mathrm{~mL}$ de metanol (Tedia, USA) 50\% e $10 \mathrm{~mL}$ de acetona (Tedia, USA) 70\%, e centrifugada em seguida.

Após a centrifugação, os sobrenadantes foram recolhidos em balão volumétrico e o volume completado com água. Em seguida, $30 \mu \mathrm{L}$ do extrato do suco de tangerina foram adicionados em tubo de ensaio contendo 3,0 $\mathrm{mL}$ da solução do radical ABTS +. .

O decréscimo da absorvância a 734 nm foi medido em intervalos de 6 min e os resultados foram expressos como capacidade antioxidante equivalente ao Trolox ${ }^{\circledR}$ (TEAC - trolox equivalent antioxidant capacity) em $\mu \mathrm{mol} . \mathrm{g}^{-1}$ de amostra.

\subsection{Análise estatística}

A análise de variância dos resultados foi feita no programa estatístico XLSTAT/Excel ${ }^{\circledast}$ e pelo teste de Tukey a 5\% de significância, para verificar a diferença entre médias. Uma segunda análise de variância foi realizada no pacote de estatística industrial do programa Statistica da StatSoft (STATISTICA, 2004), por meio de um ensaio experimental completo $2^{2}$ com três repetições no ponto central e quatro pontos axiais, analisando-se a influência das variáveis independentes temperatura e tempo, e a interação entre as mesmas na concentração dos compostos de interesse. 
Efeito do tratamento térmico na concentração de carotenoides, compostos fenólicos, ácido ascórbico e capacidade antioxidante do suco de tangerina murcote

DUTRA, A. S. et al.

\section{Resultados e discussão}

Neste trabalho, verificou-se que a $\beta$-criptoxantina foi o carotenoide presente em maior concentração no suco in natura, seguida por $\beta$-caroteno, luteína e zeaxantina (Tabela 1). De forma geral, os valores para caratenóides totais e para cada carotenoide sofreram reduções em função das condições do tratamento térmico quando comparados aos valores do suco in natura. Em alguns tratamentos, o teor de caratenóides totais aumentou como resultado de uma possível concentração do suco, dentro do trocador de calor, causada pela evaporação da água durante o tratamento térmico.

Wang et al. (2007) relataram, em estudo realizado com o fruto de tangerina murcote in natura, a presença dos caratenóides luteína $\left(76,6 \mu \mathrm{g} .100 \mathrm{~g}^{-1}\right)$, zeaxantina $\left(294,0 \mu \mathrm{g} .100 \mathrm{~g} \mathrm{~g}^{-1}\right)$, $\beta$-criptoxantina $\left(667,0 \mu \mathrm{g} .100 \mathrm{~g}^{-1}\right)$ e $\beta$-caroteno $\left(377,0 \mu \mathrm{g} .100 \mathrm{~g}^{-1}\right)$, sendo os resultados expressos em base seca. Os teores de zeaxantina e $\beta$-caroteno, obtidos neste estudo, foram menores quando comparados aos resultados descritos por Wang et al. (2007), porém, os teores de luteína e $\beta$-criptoxantina foram similares.

Stewart (1977) encontrou para o suco in natura de tangerina murcote valores de $85,5 \mu \mathrm{g} .100 \mathrm{~mL}^{-1}$ (luteína),

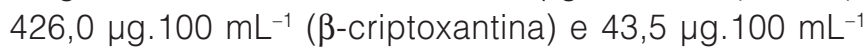
( $\beta$-caroteno).

Em quatro tratamentos, observou-se um aumento do teor de luteína causado, provavelmente, pela isomerização da zeaxantina, visto que esses caratenóides mostram entre si uma relação de isomeria estrutural. Entretanto, esses aumentos não foram estatisticamente distintos do valor encontrado no suco in natura. Apenas uma redução significativa de $42,9 \%$ do teor de zeaxantina foi observada e ocorreu no binômio $90^{\circ} \mathrm{C} / 20$. Para o teor de $\beta$-criptoxantina. apenas duas reduções significativas de 37,5 e $21 \%$ foram verificadas nos binômios $90^{\circ} \mathrm{C} / 20 \mathrm{e}$ $94^{\circ} \mathrm{C} / 30 \mathrm{~s}(\mathrm{I})$, respectivamente. Dentre os onze tratamentos aplicados, nenhum alterou de forma significativa a concentração de $\beta$-caroteno.

Gama e Sylos (2007) também observaram que os tratamentos térmicos do suco de laranja valencia, realizados em uma região de observação de 95 a $105^{\circ} \mathrm{C}$ durante $10 \mathrm{~s}$, promoveram a redução dos teores de luteína, $\beta$-caroteno e zeaxantina.

Os caratenóides apresentam estabilidades químicas distintas quanto à degradação em função das condições aplicadas durante o tratamento térmico (GAMA e SYLOS, 2007). Dentre os principais fatores que promovem a degradação dos caratenóides, seja por isomerização e/ou oxidação, podem-se citar: calor, luz, ação enzimática, oxidação promovida por peróxidos provenientes da oxidação lipídica, etc. (RODRIGUEZAMAYA, 2001).

Os resultados obtidos neste estudo são concordantes com os dados da literatura na qual são descritos, para outras espécies do gênero Citrus: as xantofilas ( $\beta$-criptoxantina, luteína e zeaxantina) e o caroteno ( $\beta$-caroteno) como sendo os caratenóides majoritários encontrados nos sucos cítricos (GAMA e SYLOS, 2005; MELÉNDEZ-MARTíNEZ et al., 2007).

Os caratenóides são compostos derivados do ácido mevalônico e produzidos pelo metabolismo secundário vegetal, cuja rota de biossíntese segue uma intrincada sequência de etapas enzimáticas. Esse fluxo biossintético pode ser afetado diretamente por uma série de fatores externos, tais como: disponibilidade de nutrientes, tipo de solo, estresse hídrico, temperatura, incidência de luz solar, época de cultivo e período de colheita, nível de maturação vegetal, etc. Dessa forma,

Tabela 1. Teores dos caratenóides presentes nos sucos in natura e tratados termicamente de tangerina murcote.

\begin{tabular}{|c|c|c|c|c|c|}
\hline $\begin{array}{c}\text { Temperatura } \\
\text { e Tempo }\end{array}$ & $\begin{array}{l}\text { Caratenóides Totais } \\
\qquad\left(\mu \mathrm{g} .100 \mathrm{~g}^{-1}\right)^{\S \ddagger}\end{array}$ & $\begin{array}{c}\text { Luteína } \\
\left(\mu \mathrm{g} .100 \mathrm{~g}^{-1}\right)^{\S}\end{array}$ & $\begin{array}{l}\text { Zeaxantina } \\
\left(\mu \mathrm{g} .100 \mathrm{~g}^{-1}\right)^{\S}\end{array}$ & $\begin{array}{c}\beta \text {-Criptoxantina } \\
\left(\mu \mathrm{g} .100 \mathrm{~g}^{-1}\right)^{\S}\end{array}$ & $\begin{array}{l}\beta \text {-Caroteno } \\
\left(\mu \mathrm{g} .100 \mathrm{~g}^{-1}\right)^{\S}\end{array}$ \\
\hline In natura & $1610,0 \pm 42,43^{b}$ & $83,0 \pm 2,83^{a b c}$ & $70,0 \pm 0,0^{a}$ & $667,0 \pm 21,21^{a}$ & $96,5 \pm 4,95^{a}$ \\
\hline $90^{\circ} \mathrm{C} ; 20 \mathrm{~s}$ & $1281,3 \pm 17,68^{e}$ & $40,0 \pm 5,66^{d}$ & $30,0 \pm 15,56^{b}$ & $416,5 \pm 36,06^{c}$ & $67,5 \pm 3,54^{a}$ \\
\hline $90^{\circ} \mathrm{C} ; 40 \mathrm{~s}$ & $1297,6 \pm 14,85^{\text {de }}$ & $74,0 \pm 7,07^{\mathrm{abcd}}$ & $60,0 \pm 8,49^{a b}$ & $549,0 \pm 21,21^{a b}$ & $80,0 \pm 1,41^{a}$ \\
\hline $98^{\circ} \mathrm{C} ; 20 \mathrm{~s}$ & $1342,7 \pm 2,83^{\text {cde }}$ & $70,0 \pm 11,31^{\mathrm{abcd}}$ & $71,5 \pm 2,12^{\mathrm{a}}$ & $591,0 \pm 43,84^{a b}$ & $84,5 \pm 14,85^{a}$ \\
\hline $98^{\circ} \mathrm{C} ; 40 \mathrm{~s}$ & $1328,1 \pm 51,62^{\text {cde }}$ & $83,5 \pm 16,26^{a b c}$ & $66,5 \pm 10,61^{a}$ & $614,0 \pm 16,97^{a b}$ & $82,5 \pm 6,36^{a}$ \\
\hline $94^{\circ} \mathrm{C} ; 30 \mathrm{~s}(\mathrm{I})$ & $1426,4 \pm 35,36^{c d}$ & $55,0 \pm 2,12^{c d}$ & $50,5 \pm 2,12^{\mathrm{ab}}$ & $527,5 \pm 7,78^{\mathrm{bc}}$ & $87,0 \pm 2,83^{a}$ \\
\hline $94^{\circ} \mathrm{C} ; 30 \mathrm{~s}(\mathrm{II})$ & $1643,6 \pm 24,04^{a b}$ & $82,0 \pm 15,56^{a b c}$ & $66,5 \pm 17,68^{a}$ & $633,5 \pm 26,16^{a b}$ & $94,0 \pm 2,83^{a}$ \\
\hline $94^{\circ} \mathrm{C} ; 30 \mathrm{~s}(\mathrm{III})$ & $1443,2 \pm 48,79^{c}$ & $97,0 \pm 12,73^{a}$ & $53,0 \pm 8,49^{a b}$ & $587,5 \pm 13,44^{a b}$ & $93,5 \pm 3,54^{a}$ \\
\hline $94^{\circ} \mathrm{C} ; 16 \mathrm{~s}$ & $1562,7 \pm 15,56^{a b}$ & $104 \pm 2,83^{a}$ & $55,0 \pm 1,41^{a b}$ & $610,0 \pm 42,43^{a b}$ & $92,5 \pm 6,36^{a}$ \\
\hline $94^{\circ} \mathrm{C} ; 44 \mathrm{~s}$ & $1760,4 \pm 51,62^{a}$ & $90,0 \pm 2,83^{a b}$ & $54,5 \pm 4,95^{\mathrm{ab}}$ & $662,5 \pm 51,62^{a}$ & $86,0 \pm 22,63^{a}$ \\
\hline $88^{\circ} \mathrm{C} ; 30 \mathrm{~s}$ & $1420,4 \pm 24,75^{\text {cde }}$ & $99,0 \pm 1,41^{a}$ & $60,5 \pm 0,71^{\mathrm{ab}}$ & $632,5 \pm 6,36^{a b}$ & $93,5 \pm 2,12^{a}$ \\
\hline $100^{\circ} \mathrm{C} ; 30 \mathrm{~s}$ & $1129,8 \pm 32,53^{\dagger}$ & $60,0 \pm 7,07^{\mathrm{bcd}}$ & $45,5 \pm 0,71^{\mathrm{ab}}$ & $566,5 \pm 45,96^{a b}$ & $83,5 \pm 9,19^{a}$ \\
\hline
\end{tabular}

Letras diferentes na mesma coluna evidenciam diferença significativa no nível de $5 \%$ de significância pelo teste de Tukey. §Valores expressos

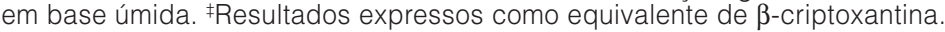


Efeito do tratamento térmico na concentração de carotenoides, compostos fenólicos, ácido ascórbico e capacidade antioxidante do suco de tangerina murcote

DUTRA, A. S. et al.

os caratenóides presentes em uma dada matriz podem apresentar diferenças no perfil químico assim como na concentração, quando são feitas comparações diretas entre trabalhos distintos.

A partir dos gráficos de Pareto para cada carotenoide analisado (Figura 1), pode-se constatar que os resultados obtidos não foram afetados de forma significativa pelas variáveis tempo e temperatura, assim como pela interação entre as mesmas, pois os valores do efeito estimado padronizado para essas variáveis, com base nos modelos linear e quadrático, não ultrapassaram a linha de significância $(p=0,05)$.

Os dados de concentração de ácido ascórbico, compostos fenólicos totais e capacidade antioxidante estão descritos na Tabela 2. O teor de ácido ascórbico apresentou uma redução significativa de $27 \%$, quando comparado ao suco in natura, somente para o binômio $100{ }^{\circ} \mathrm{C} / 30$ s. Para os demais tratamentos, as alterações
Gráfico de pareto dos efeitos padronizados Variável: carotenóides totais

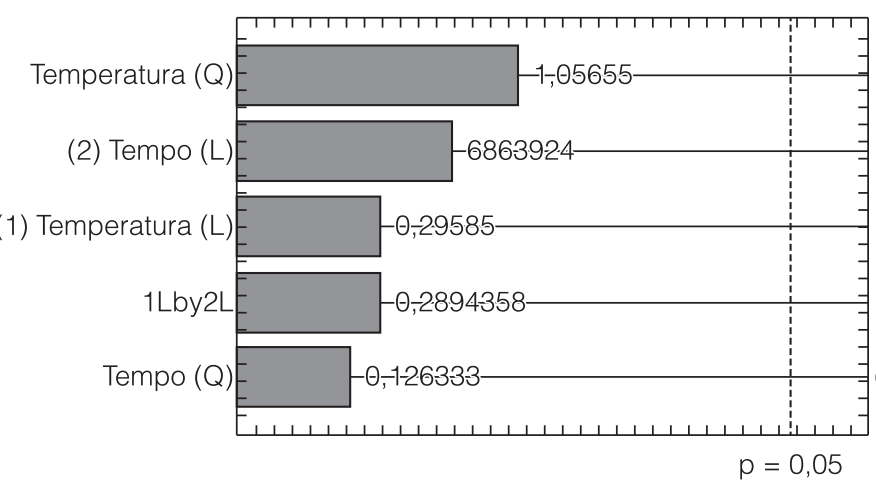

Efeito estimado padronizado (valor absoluto)

Gráfico de pareto dos efeitos padronizados Variável: zeaxantina

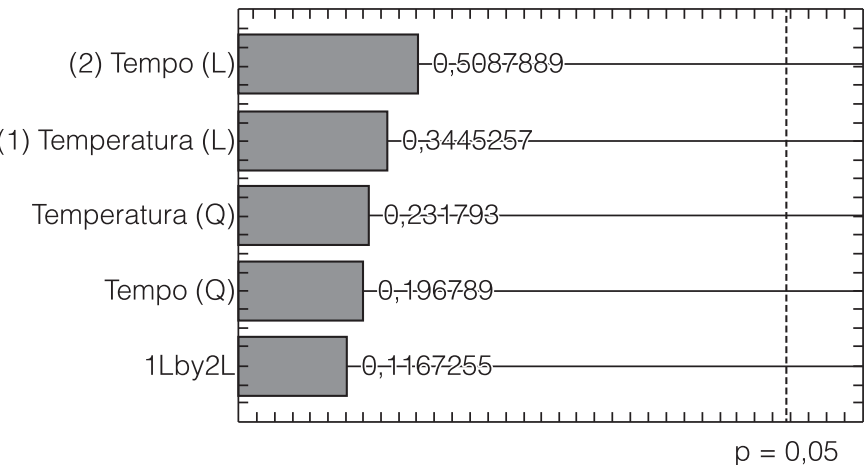

Efeito estimado padronizado (valor absoluto)
Gráfico de pareto dos efeitos padronizados Variável: luteína

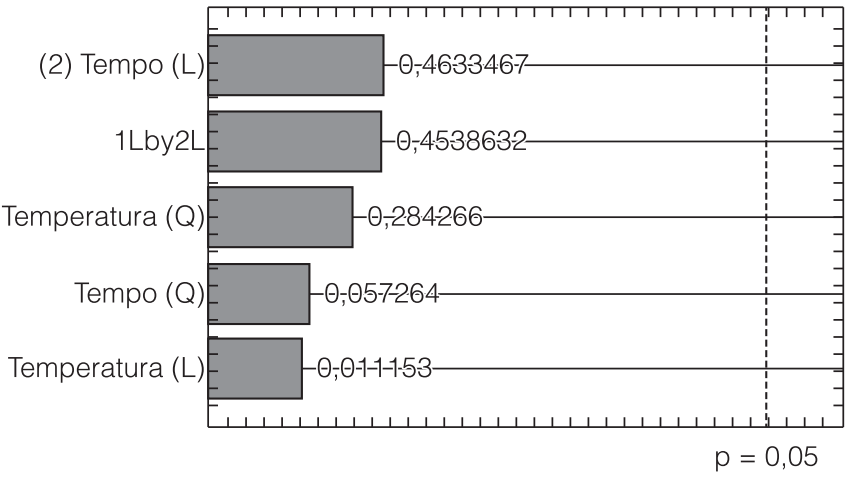

Efeito estimado padronizado (valor absoluto)

Gráfico de pareto dos efeitos padronizados Variável: $\beta$-criptoxantina

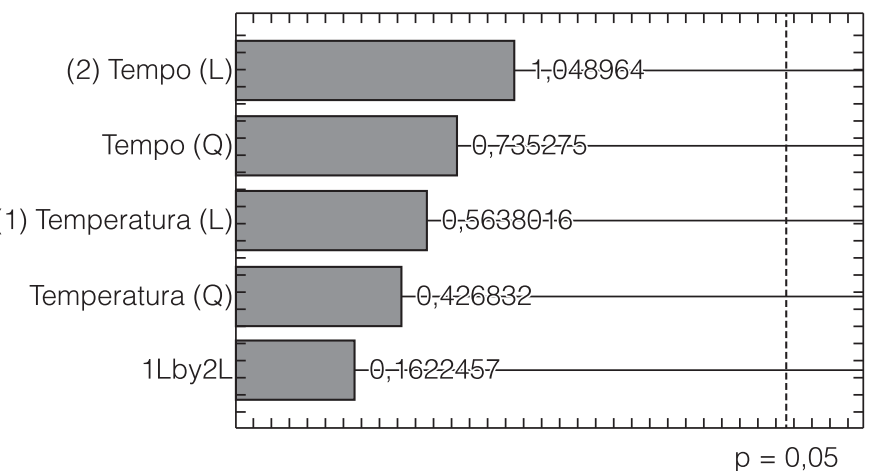

Efeito estimado padronizado (valor absoluto)

Gráfico de pareto dos efeitos padronizados

Variável: $\beta$-caroteno

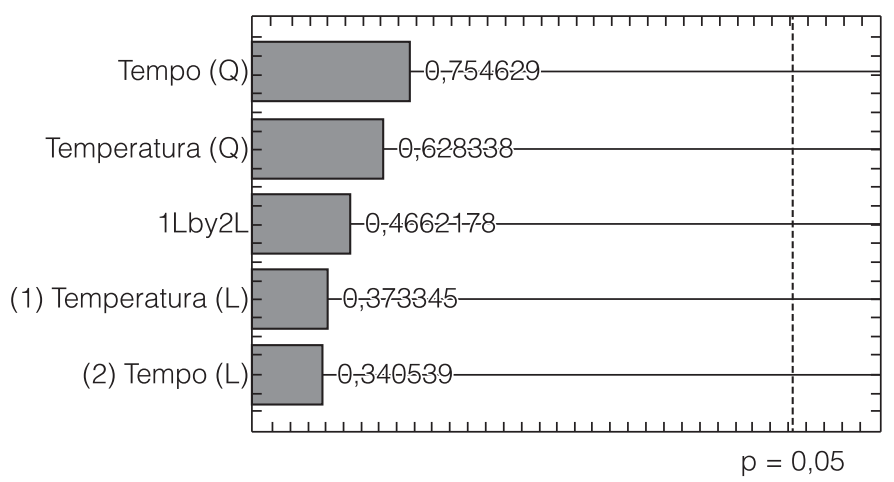

Efeito estimado padronizado (valor absoluto)

Figura 1. Gráficos de Pareto para caratenóides totais, luteína, zeaxantina, $\beta$-criptoxantina e $\beta$-caroteno em função das variáveis independentes temperatura e tempo. 
Efeito do tratamento térmico na concentração de carotenoides, compostos fenólicos, ácido ascórbico e capacidade antioxidante do suco de tangerina murcote

DUTRA, A. S. et al.

observadas nos teores não foram significativas. Estudos têm demonstrado que o uso do calor pode promover alterações na concentração de ácido ascórbico, assim como em outras propriedades físico-químicas do suco (MELÉNDEZ-MARTÍNEZ et al., 2007; FARNWORTH et al., 2001; NAGY, 1980).

A análise de variância (ANOVA) indicou que os resultados obtidos para ácido ascórbico sofreram de forma significativa a influência da variável independente temperatura, pois se observa pelo gráfico de Pareto (Figura 2) que a linha de significância $(p=0,05)$ foi ultrapassada. O comportamento de degradação observado foi descrito por um modelo quadrático e a maior redução foi observada para o tratamento realizado na temperatura mais elevada $\left(100^{\circ} \mathrm{C} / 30 \mathrm{~s}\right)$.

Para os compostos fenólicos totais, observou-se um aumento dos teores quando o suco foi tratado termicamente a $88^{\circ} \mathrm{C} / 30 \mathrm{~s}, 94^{\circ} \mathrm{C} / 16 \mathrm{~s}, 94^{\circ} \mathrm{C} / 44 \mathrm{~s}$,

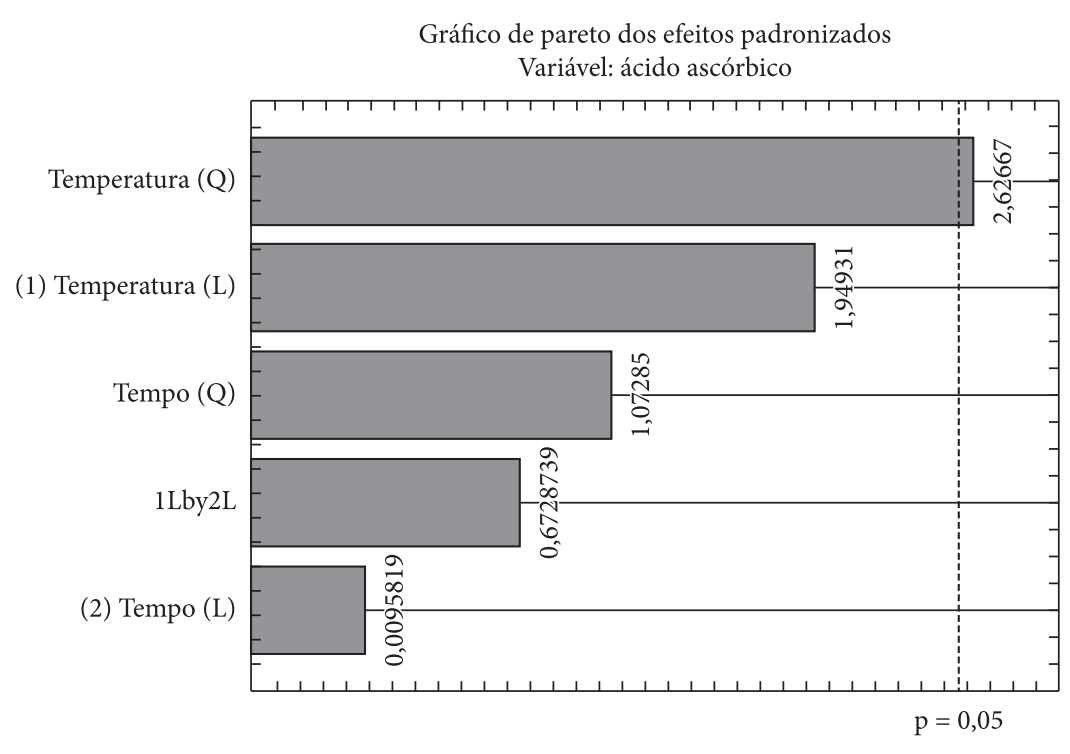

Efeito estimado padronizado (valor absoluto)

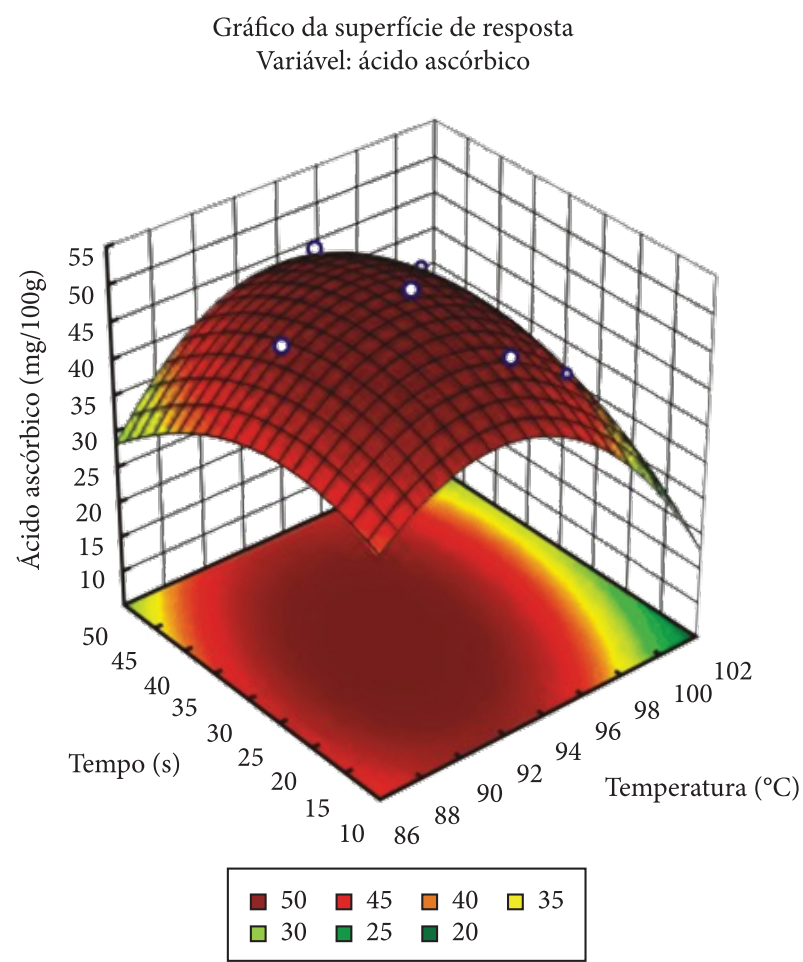

Figura 2. Gráfico de Pareto e superfície de resposta para ácido ascórbico em função das variáveis independentes temperatura e tempo. 
Efeito do tratamento térmico na concentração de carotenoides, compostos fenólicos, ácido ascórbico e capacidade antioxidante do suco de tangerina murcote

DUTRA, A. S. et al.

$98{ }^{\circ} \mathrm{C} / 20$ s e $100{ }^{\circ} \mathrm{C} / 30 \mathrm{~s}$ (Tabela 2). Tais aumentos podem ser o resultado da uma provável redução do teor de água presente no suco em razão da evaporação ocorrida dentro do trocador de calor. No entanto, essas variações não foram significativas quando comparadas ao suco in natura.

Para a capacidade antioxidante, foram observadas reduções significativas na maioria das condições de tratamento térmico avaliadas (Tabela 2). A maior redução (34\%) ocorreu no binômio $100{ }^{\circ} \mathrm{C} / 30 \mathrm{~s}$, assim como observado para o ácido ascórbico. Dessa forma, a redução da capacidade antioxidante está relacionada diretamente, entre outros fatores, à redução da concentração do ácido ascórbico.

Os resultados obtidos para compostos fenólicos totais e capacidade antioxidante não apresentaram efeitos significativos das variáveis independentes temperatura e tempo de pasteurização, assim como da interação entre essas variáveis, pois nos gráficos de Pareto (Figura 3) não se ultrapassou a linha de significância $(p=0,05)$.

A maioria dos binômios avaliados não alterou de forma significativa as características físico-químicas do suco tratado termicamente, quando comparado ao in natura, para compostos fenólicos totais, $\beta$-caroteno, luteína e zeaxantina (exceto no binômio $90{ }^{\circ} \mathrm{C} / 20 \mathrm{~s}$ ), e ácido ascórbico (exceto no binômio $100{ }^{\circ} \mathrm{C} / 30 \mathrm{~s}$ ). A capacidade antioxidante apresentou uma redução significativa em $82 \%$ dos binômios testados, sendo mais intensa no binômio $100{ }^{\circ} \mathrm{C} / 30 \mathrm{~s}$.

Para caratenóides totais, apenas dois dos binômios testados não causaram alterações significativas nos teores em comparação ao suco in natura. Porém, a análise estatística mostrou que tais variações não foram resultado da temperatura e do tempo aplicados no estudo, assim como das interações entre essas variáveis.

Tabela 2. Teores de ácido ascórbico, compostos fenólicos totais e capacidade antioxidante dos sucos in natura e tratado termicamente de tangerina murcote.

\begin{tabular}{|c|c|c|c|}
\hline $\begin{array}{c}\text { Temperatura } \\
\text { e tempo }\end{array}$ & $\begin{array}{c}\text { Ácido ascórbico } \\
\left(\mathrm{mg} .100 \mathrm{~g}^{-1}\right)\end{array}$ & $\begin{array}{c}\text { Fenólicos Totais } \\
\text { (mg ácido gálico.100 } \mathrm{g}^{-1} \text { ) }\end{array}$ & $\begin{array}{c}\text { Capacidade antioxidante } \\
\left(\mu \mathrm{mol} \text { Trolox } \cdot \mathrm{g}^{-1}\right)\end{array}$ \\
\hline In natura & $50,87 \pm 0,27^{a}$ & $52,32 \pm 5,97^{a}$ & $3,27 \pm 0,11^{a}$ \\
\hline $90^{\circ} \mathrm{C} ; 20 \mathrm{~s}$ & $44,13 \pm 5,87^{\mathrm{ab}}$ & $44,48 \pm 7,06^{a}$ & $2,34 \pm 0,30^{b c}$ \\
\hline $90^{\circ} \mathrm{C} ; 40 \mathrm{~s}$ & $42,28 \pm 2,88^{a b}$ & $48,40 \pm 9,43^{a}$ & $3,02 \pm 0,10^{a b}$ \\
\hline $98^{\circ} \mathrm{C} ; 20 \mathrm{~s}$ & $40,50 \pm 2,81^{\mathrm{ab}}$ & $53,34 \pm 13,99^{a}$ & $2,27 \pm 0,17^{b c}$ \\
\hline $98^{\circ} \mathrm{C} ; 40 \mathrm{~s}$ & $43,94 \pm 2,78^{\mathrm{ab}}$ & $51,58 \pm 6,69^{a}$ & $2,42 \pm 0,07^{b c}$ \\
\hline $94^{\circ} \mathrm{C} ; 30 \mathrm{~s} \mathrm{I}$ & $50,98 \pm 3,00^{a}$ & $44,81 \pm 2,79^{a}$ & $2,51 \pm 0,11^{b c}$ \\
\hline $94^{\circ} \mathrm{C} ; 30 \mathrm{~s} I I$ & $51,00 \pm 2,76^{a}$ & $51,60 \pm 9,25^{a}$ & $2,26 \pm 0,32^{c}$ \\
\hline $94^{\circ} \mathrm{C} ; 30 \mathrm{~s} \mathrm{III}$ & $50,89 \pm 3,17^{a}$ & $45,94 \pm 6,94^{a}$ & $2,27 \pm 0,40^{\circ}$ \\
\hline $94^{\circ} \mathrm{C} ; 16 \mathrm{~s}$ & $50,16 \pm 3,10^{a}$ & $56,72 \pm 4,33^{a}$ & $2,38 \pm 0,21^{b c}$ \\
\hline $94^{\circ} \mathrm{C} ; 44 \mathrm{~s}$ & $49,10 \pm 5,93^{a}$ & $56,83 \pm 7,54^{a}$ & $2,25 \pm 0,15^{c}$ \\
\hline $88^{\circ} \mathrm{C} ; 30 \mathrm{~s}$ & $50,77 \pm 2,86^{a}$ & $54,99 \pm 2,07^{a}$ & $2,70 \pm 0,26^{a b c}$ \\
\hline $100{ }^{\circ} \mathrm{C} ; 30 \mathrm{~s}$ & $37,19 \pm 2,90^{b}$ & $60,70 \pm 3,57^{a}$ & $2,16 \pm 0,07^{c}$ \\
\hline
\end{tabular}

Letras diferentes na mesma coluna evidenciam diferença significativa no nível de $5 \%$ de significância pelo teste de Tukey.

Gráfico de pareto dos efeitos padronizados Variável: capacidade antioxidante

(1) Temperatura (L)

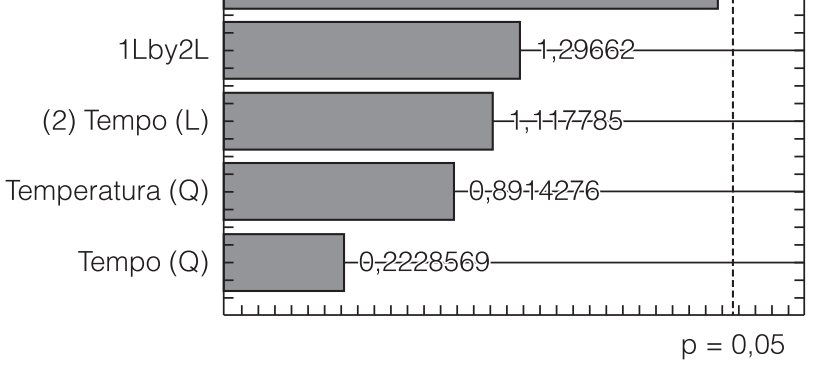

Efeito estimado padronizado (valor absoluto)
Gráfico de pareto dos efeitos padronizados Variável: compostos fenólicos totais

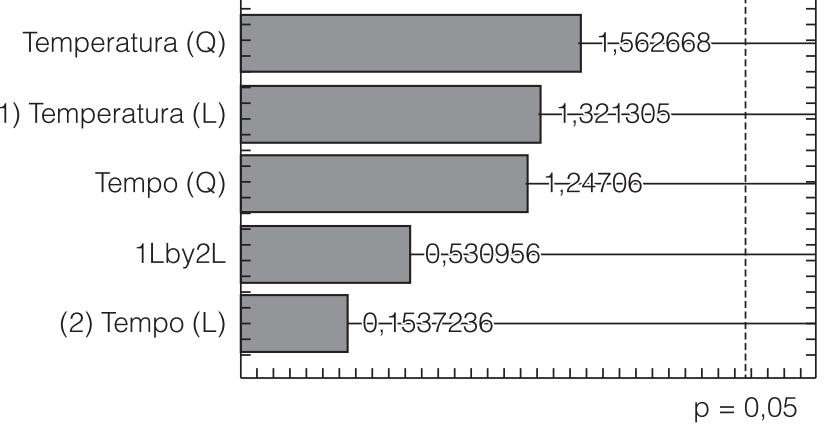

Efeito estimado padronizado (valor absoluto)

Figura 3. Gráficos de Pareto para compostos fenólicos totais e capacidade antioxidante em função das variáveis independentes temperatura e tempo. 
Efeito do tratamento térmico na concentração de carotenoides, compostos fenólicos, ácido ascórbico e capacidade antioxidante do suco de tangerina murcote

DUTRA, A. S. et al.

Os principais fatores responsáveis pela diminuição dos teores dos caratenóides são o oxigênio atmosférico e a luz ambiente, aos quais estiveram expostos ao longo do processo, propiciando assim sua foto-oxidação.

\section{Conclusões}

As variáveis independentes temperatura e tempo, e a interação das mesmas não influenciaram de maneira significativa os resultados obtidos para as características avaliadas, com exceção do ácido ascórbico, no qual a influência da temperatura foi significativa na redução da concentração. O tratamento a $100^{\circ} \mathrm{C}$ apresentou o maior valor de redução do ácido ascórbico quando comparado ao valor determinado para o suco in natura.

Não se observou, dentre todos os binômios aplicados, aquele que apresentasse o mesmo comportamento para todas as características avaliadas, pois os binômios exerceram influências distintas em cada uma das características. Porém, os tratamentos térmicos realizados a $94{ }^{\circ} \mathrm{C}$ por 16 a $44 \mathrm{~s}$ possibilitaram menores alterações e/ou maiores retenções nos compostos determinados.

\section{Referências}

AZEVEDO, F. A.; PIO, R. M. Influência da polinização sobre o número de sementes do tangor "Murcote". Revista Brasileira de Fruticultura, Cruz das Almas, v. 24, n. 2, p. 468-471, 2002. http://dx.doi.org/10.1590/S0100-29452002000200037

BENASSI, M. D.; ANTUNES, A. J. A comparison of metaphosphoric and oxalic acids as extractants solutions for the determination of vitamin $C$ in selected vegetables. Arquivos de Biologia e Tecnologia, Curitiba, v. 31, n. 4, p. 507-503, 1998.

CANO, A.; MEDINA, A.; BERNEJO, A. Bioactive compounds in different Citrus varieties: discrimination among cultivars. Journal of Food Composition and Analysis, San Diego, v. 21, n. 5 , p. 377-381, 2008. http://dx.doi.org/10.1016/j.jfca.2008.03.005

CASER, D. V.; AMARO, A. A. Tangerina: tendências no cultivo no Estado de São Paulo. Análises e Indicadores do Agronegócio, São Paulo, v. 1, n. 12, p. 1-7, 2006.

DUGO, P.; HERRERO, M.; KUMM, T.; GIUFFRIDA, D.; DUGO, G.; MONDELLO, L. Comprehensive normal phase x reversed phase liquid chromatography coupled to photodiode array and mass spectrometry detection for the analysis of free carotenoids and carotenoid esters from mandarin. Journal of Chromatography A. Amsterdam, v. 1189, n. 1-2, p. 196-206, 2008. http://dx.doi. org/10.1016/j.chroma.2007.11.116

FARNWORTH, E. R.; LAGACÉ, M.; COUTURE, R.; YAYLAYAN, V.; STEWART, B. Thermal processing, storage conditions, and the composition and physical properties of orange juice. Food Research International, Barking, v. 34, n. 1, p. 25-30, 2001. http://dx.doi.org/10.1016/S0963-9969(00)00124-1
FIGUEIREDO, J. O.; NEGRI, J. D.; MATTOS JUNIOR, D.; PIO, R. M.; AZEVEDO, F. A.; GARCIA, V. X. P. Comportamento de 16 porta-enxertos para o tangor Murcott na região de Itirapina-SP. Revista Brasileira de Fruticultura, Cruz das Almas, v. 28, n. 1, p. 76-78, 2006. http://dx.doi.org/10.1590/ S0100-29452006000100022

GAMA, J. J. T.; SYLOS, C. M. Major carotenoid composition of Brazilian Valencia orange juice: identification and quantification by HPLC. Food Research International, London, v. 38, p. 899-903, 2005. http://dx.doi.org/10.1016/j.foodres.2005.03.008

GAMA, J. J. T.; SYLOS, C. M. Effect of thermal pasteurization and concentration on carotenoid composition of Brazilian Valencia orange juice. Food Chemistry, London, v. 100, n. 4, p. 1686-1690, 2007. http://dx.doi.org/10.1016/j. foodchem.2005.01.062

GEORGÉ, S.; BRAT, P.; ALTER, P.; AMIOT, M. J. Rapid determination of polyphenols and vitamin $C$ in plant derived products. Journal of Agricultural and Food Chemistry, Easton, v. 53, n. 5, p. 1370-1373, 2005. http://dx.doi.org/10.1021/ jf048396b

GOODNER, K. L.; ROUSEFF, R. L.; HOFSOMMER, H. J. Orange, mandarin, and hybrid classification using multivariate statistics based on carotenoid profiles. Journal of Agricultural and Food Chemistry, Easton, v. 49, n. 3, p. 1146-1150, 2001. http://dx.doi. org/10.1021/jf000866o

HORWITZ, W. (Ed.). Official Methods of Analysis of the Association of Official Analytical Chemists. 17th ed. Gaithersburg: AOAC, 2000. 2 v.

LEACH, G.; OLIVEIRA, G.; MORAIS, R. Production of a carotenoid rich product by alginate entrapment and fluid-bed drying of Dunaliella salina. Journal of the Science of Food and Agriculture, London, v. 76, n. 2, p. 298-302, 1998. http:// dx.doi.org/10.1002/(SICI)1097-0010(199802)76:2<298::AIDJSFA953>3.0.CO;2-0

MELÉNDEZ-MARTínEZ, A. J.; VICARIO, I. M.; HEREDIA, F. $J$. Provitamin A carotenoids and ascorbic acid contents of the different types of orange juices marketed in Spain. Food Chemistry, London, v. 101, n. 1, p. 177-184, 2007. http://dx. doi. org/10.1016/j.foodchem.2006.01.023

NAGY, S. Vitamin C contents of citrus fruit and their products: a review. Journal of Agricultural and Food Chemistry, Easton v. 28, n. 1, p. 8-18, 1980. http://dx.doi.org/10.1021/j60227a026

OLSON, J. A. Benefits and liabilities of vitamin A and carotenoids. Journal of Nutrition, Philadelphia, v. 126, n. 4, p. S1208S1212, 1996.

OMONI, A. O.; ALUKO, R. E. The anti-carcinogenic and antiatherogenic effects of lycopene: a review. Trends in Food Science \& Technology, Cambridge, v. 16, n. 8, p. 344-350, 2005. http://dx.doi.org/10.1016/j.tifs.2005.02.002 
Efeito do tratamento térmico na concentração de carotenoides, compostos fenólicos, ácido ascórbico e capacidade antioxidante do suco de tangerina murcote

DUTRA, A. S. et al.

PACHECO, S. Preparo de Padrões Analíticos, Estudo de Estabilidade e Parâmetros de Validação para Ensaio de Carotenóides por Cromatografia Líquida. 2009. 106 f. Dissertação (Mestrado em Ciência e Tecnologia de Alimentos)Instituto de Tecnologia, Universidade Federal Rural do Rio de Janeiro, Rio de Janeiro, 2009.

RE, R.; PELlegrinI, N.; PROTEGgente, A.; PANNAlA, A.; YANG, M.; RICE-EVANS, C. Antioxidant activity applying an improved ABTS radical cation decolorization assay. Free Radical Biology \& Medicine, New York, v. 26, n. 9-10, p. 1231-1237, 1999. http://dx.doi.org/10.1080/14786419.201 0.530267

RIVAS, A.; RODRIGO, D.; MARTÍNEZ, A.; BARBOSA-CÁNOVAS, G. V.; RODRIGO, M. Effect of PEF and heat pasteurization on the physical-chemical characteristics of blended orange and carrot juice. LWT - Food Science and Technology, London, v. 39 , n. 10 , p. 1163-1170, 2006. http://dx.doi.org/10.1016/j. Iwt.2005.07.002

RODRIGUEZ-AMAYA, D. B. A Guide to Carotenoid Analysis in Foods. Washington: ILSI Press, 2001. 64 p.

RUFINO, M. S. M.; ALVES, R. E.; BRITO, E. S.; MORAIS, S. M.; SAMPAIO, C. G.; PÉREZ-JIMÉNEZ, J.; SAURA-CALIXTO,
F. D. Metodologia Científica: Determinação da Atividade Antioxidante Total em Frutas pela Captura do Radical Livre ABTS+. Fortaleza: Embrapa Agroindústria Tropical, 2007. 4 p.

SINGLETON, V. L.; ROSSI JUNIOR, R. J. Colorimetry of total phenolics with phosphomolybdic-phosphotungstic acid reagents. American Journal of Enology and Viticulture, Davis, v. 16, n. 3, p. 144-158, 1965.

SNODDERLY, D. M. Evidence for protection against age-related macular degeneration by carotenoids and antioxidant vitamins. American Journal of Clinical Nutrition, New York, v. 62, n. 6 , p. S1448-S1461, 1995.

STATISTICA. Data Analysis Software System. version 7. Tulsa: StatSoft, 2004. 1 CD-ROM.

STEWART, I. Provitamin A and carotenoid content of Citrus juices. Journal of Agricultural and Food Chemistry, Easton, v. 25, n. 5, p. 1132-1137, 1977. http://dx.doi.org/10.1021/jf60213a048 WANG, Y.; CHUENG, Y.; KU, Y. Quantitation of bioactive compounds in Citrus fruits cultivated in Taiwan. Food Chemistry, London, v. 102, n. 4, p. 1163-1171, 2007. http://dx.doi. org/10.1016/j.foodchem.2006.06.057 